\title{
Efecto de la pre-medicación con alprazolam por vía oral sobre la presión arterial en pacientes hipertensos en cirugías electivas: un ensayo clínico
}

\author{
Walter Delgado Maidana, Evanhy Vega Carduz \\ Departamento Central de Anestesiología, FCM UNA. Paraguay \\ Cómo referenciar este artículo/ \\ How to reference this article: \\ Delgado W, Vega E. Efecto de la pre-medicación \\ con alprazolam por vía oral sobre la presión arterial \\ en pacientes hipertensos en cirugías electivas: un \\ ensayo clínico. Mem. Inst. Investig. Cienc. Salud. \\ $2016 ; 14(3): 34-43$
}

\section{R E S U M E N}

Los ansiolíticos como el alprazolam podrían colaborar en mantener la presión arterial en pacientes hipertensos que deberán someterse a cirugía. El objetivo fue evaluar eficacia de la pre-medicación con alprazolam vía oral para mantener estables cifras de presión arterial en hipertensos programados para cirugías. Ensayo clínico aleatorizado, controlado y a doble ciego. Los pacientes fueron asignados al grupo que recibió alprazolam como premedicación (Grupo A) o al grupo control (Grupo P) que no lo recibió. Se registraron la variación de la presión arterial media (PAM), frecuencia cardíaca (FC) y frecuencia respiratoria (FR) entre el día previo y el día de la cirugía. En el Grupo A se encontró un aumento promedio de la PAM de $0,27 \mathrm{mmHg}$ y en el grupo $\mathrm{P}$ de $7,52 \mathrm{mmHg}(\mathrm{p}=0,0035)$. La variación promedio de la FC fue de 2,13 latidos por minuto en el Grupo A y de 5,38 latidos por minuto en el Grupo P ( $p=$ $0,0008)$; y de la frecuencia respiratoria fue de $-2,29$ respiraciones por minuto en el Grupo $A$ y de $-1,18$ respiraciones por minuto en el Grupo $P(p=0,206)$. Se halló un aumento significativo de la PAM y la FC en el Grupo P con respecto al Grupo A, no así de la FR. La administración del alprazolam como pre-medicación operatoria evita aumentos significativos de la presión arterial y de la frecuencia cardíaca en los pacientes hipertensos.

Palabras clave: alprazolam, ansiedad, periodo preoperatorio, presión arterial.

\section{Effect of premedication with alprazolam orally administered on blood pressure in hypertensive patients in elective surgeries: a clinical trial}

\section{A B S T RACT}

Anxiolytics such as alprazolam may help to maintain blood pressure in hypertensive patients who undergo surgery. The aim was to evaluate efficacy of pre-medication with alprazolam orally administered to maintain stable blood pressure in hypertensives programmed for surgeries. Randomized, controlled, double-blind clinical trial. Patients were assigned to the group that received alprazolam as premedication (Group A) or to the control group (Group P) which did not receive it. The variation of the mean arterial pressure (MAP), heart rate (HR) and respiratory rate (RR) were recorded between the day before and the day of the surgery. In Group A, we found an average increase in MAP of $0.27 \mathrm{mmHg}$ and in the Group $P 7.52 \mathrm{mmHg}(p=0.0035)$. The mean HR variation was 2.13 beats per minute in Group A and 5.38 beats per minute in Group P $(p=0.0008)$. The mean RR variantion was -2.29 breaths per minute in Group $A$ and -1.18 breaths per minute in Group $P(p=0.206)$. There was a significant increase in the MAP and HR in Group P compared to Group A, but not in the RR. The administration of alprazolam as an operative premedication avoids significant increases in blood pressure and heart rate in hypertensive patients.

Key words: alprazolam, anxiety, preoperative period, blood pressure. 


\section{INTRODUCCIÓN}

La hipertensión arterial (HTA) constituye una de las causas médicas más frecuentes de aplazamiento de una cirugía electiva (1). Desafortunadamente, no todos los pacientes hipertensos están tratados y entre los que siguen un tratamiento existe una proporción que no consigue un control adecuado de las cifras de presión arterial.

Existen estudios epidemiológicos que indican que la HTA se asocia a un aumento de morbimortalidad durante el período peri-operatorio. Estos pacientes pueden presentar repercusión sobre algún órgano blanco a nivel cardíaco, encefálico, renal o vascular periférico (2-4). Especialmente, la HTA es un factor de riesgo demostrado en las isquemias cerebral y cardíaca. El riesgo operatorio del paciente hipertenso está esencialmente ligado a la enfermedad coronaria por lo que en los operados hipertensos la incidencia de episodios de isquemia coronaria es mayor (5).

Las cirugías electivas constituyen cirugías que no representan urgencias o emergencias quirúrgicas, y para las cuales el paciente debería tener una óptima preparación clínica con la finalidad de reducir al mínimo el riesgo de complicaciones intra y postoperatorias.

Con frecuencia se observa en sala de operaciones pacientes que fueron programados para cirugías electivas y que presentan cifras de presión arterial muy elevadas a su ingreso a quirófano. La hipertensión preoperatoria puede estar asociada con una amplia variedad de condiciones clínicas, como ser: pacientes que no se conocen hipertensos, pacientes conocidos hipertensos que no presentan un tratamiento adecuado o aquellos que presentan una elevación anormal de la presión arterial en respuesta a situaciones estresantes, como dolor o ansiedad (5).

El acto anestésico quirúrgico genera ansiedad en el paciente, por lo que es muy importante enfocarse a disminuir la ansiedad mediante una adecuada atención hospitalaria que incluya consulta pre-anestésica oportuna con la debida preparación psicológica y farmacológica del enfermo $(6,7)$.

Por las características fisiopatológicas, en los pacientes hipertensos el comportamiento de la presión arterial es más lábil y sensible a las modificaciones por las catecolaminas que son liberadas en situaciones estresantes. En los pacientes hipertensos, la ansiedad podría contribuir en manera significativa a una elevación anormal de la presión arterial antes de empezar la cirugía (7), lo que podría incurrir en la suspensión de la cirugía programada por una crisis hipertensiva, el aumento del sangrado en el peroperatorio y postoperatorio, hasta la presentación de complicaciones cardíacas y/ o neurológicas en el peri-operatorio.

La pre-medicación ansiolítica a un paciente provoca sedación, disminuyendo la liberación de catecolaminas y atenuando el impacto emocional sobre la presión arterial, obteniéndose también una mejor cooperación del enfermo, que finalmente será un paciente satisfecho con el tratamiento y las atenciones otorgadas por el equipo de salud $(7,8)$. Además, está comprobado que la disminución de la ansiedad preoperatoria mejora la recuperación clínica postoperatoria $(9,10)$.

En algunos estudios se constató que las benzodiacepinas como el diacepam y el alprazolam fueron tan eficientes como el captopril para disminuir la presión arterial en pacientes hipertensos $(11,12)$.

En el Hospital de Clínicas, los pacientes que se someterán a cirugías electivas generalmente no reciben ansiolíticos de rutina antes de las cirugías, ya que en este centro médico, dichos fármacos no están protocolizados como pre-medicación preoperatoria.

Por lo tanto, resultaría importante conocer la implicancia de una pre-medicación oral ansiolítica con alprazolam preoperatorio en el comportamiento de la presión arterial de los pacientes hipertensos que deberán someterse a una cirugía electiva, por lo que el objetivo de esta investigación fue determinar el efecto del alprazolam sobre la presión arterial en los hipertensos programados para cirugías electivas. Los resultados de este trabajo serán de mucho valor para demostrar la importancia de instaurar una protocolización de medicación ansiolítica en el preoperatorio de pacientes hipertensos, dentro del contexto de un mejoramiento de los resultados clínicos y quirúrgicos en el postoperatorio. Con esto se minimizará la influencia de un factor importante en el periodo preoperatorio como lo es la ansiedad y que por sus efectos fisiopatológico produce una alteración de las cifras tensionales en los pacientes hipertensos, lo que puede desencadenar eventos como la suspensión de la cirugía electiva o una descompensación clínica de los pacientes. La administración protocolizada del alprazolam en el periodo preoperatorio podría contribuir a 
evitar gastos innecesarios por cirugías electivas suspendidas y a disminuir la posibilidad de complicaciones relacionadas a los aumentos de presión arterial.

\section{MATERIALES Y MÉTODO}

El protocolo de investigación fue aprobado por el Comité de Ética de Investigación de la Facultad de Ciencias Médicas (UNA).

\section{Selección de pacientes}

Se realizó un ensayo clínico aleatorizado, controlado y a doble ciego en 88 pacientes adultos con diagnóstico de hipertensión arterial a ser sometidos a cirugías electivas en el Hospital de Clínicas, Facultad de Ciencias Médicas, Universidad Nacional de Asunción (FCMUNA), desde diciembre del 2012 hasta junio del 2013. Fueron considerados para el estudio todos los pacientes con diagnóstico previo de hipertensión arterial y que estaban con medicación antihipertensiva, programados para cirugías electivas, con edad comprendida entre 35 y 65 años, y que se hayan internado como mínimo el día previo a la cirugía. Fueron excluídos los pacientes con hipertiroidismo, con feocromocitoma, consumidores de cocaína, anfetaminas o anabólicos, en los cuáles la presión arterial podría estar alterada. También a los pacientes en tratamiento ansiolítico en forma crónica con benzodiacepinas, antipsicóticos o antidepresivos, en los que el efecto de dos dosis de alprazolam podría resultar encubierto y a los pacientes cuyas cirugías electivas se iniciaron después del mediodía, ya que no se observaría el efecto clínico de la dosis de alprazolam administrada por que la vida media es de 6 a 8 hs.

\section{Aleatorización y obtención del consentimiento informado}

El muestreo de los pacientes incluidos fue probabilístico y los pacientes fueron asignados en forma aleatoria simple, a dos grupos. Un primer Grupo A: pre-medicado con 0,5 mg. de alprazolam por vía oral y un segundo Grupo P pre-medicado con $500 \mathrm{mg}$. de vitamina C como placebo. La asignación aleatoria de la pre-medicación con alprazolam o con placebo fue colocada por adelantado en una serie de sobres sellados por un investigador que no intervino en su apertura. Se elaboró una lista de los pacientes con base a la planilla de cirugías programadas del Hospital de Clínicas que se publica el día hábil anterior a la realización de las cirugías. Se contactó con los pacientes hipertensos que cumplían los criterios de inclusión y se les invitó a participar del estudio, explicándoles claramente el objetivo del estudio y se les solicitó la firma del consentimiento informado firmado. A los pacientes que aceptaron participar del estudio se les realizó una anamnesis y se procedió a la aleatorización asignándoles a uno de los de los dos grupos (Grupo A) premedicación con Alprazolam por vía oral y (Grupo P) pre-medicación con placebo mediante la apertura de un sobre que los mismos pacientes eligieron, sin que conozcan el grupo al que fue asignado. Los grupos asignados fueron anotados en una planilla entregada posteriormente a otro investigador.

\section{Variable de intervención}

Consumo o no de alprazolam como pre-medicación antes de la cirugía. Variable dicotómica. El alprazolam es un fármaco ansiolítico, el cual fue utilizado en este trabajo. Se administró al Grupo A de pacientes, alprazolam $0,5 \mathrm{mg}$ por vía oral en dos ocasiones, en la noche anterior a la cirugía (a las 22:00 horas) y en la mañana en que se realizó la cirugía (a las 6:00 horas). Al Grupo P de pacientes se le administró 500 mg de vitamina C como placebo en vez de las benzodiacepinas en las mismas condiciones y horarios.

\section{Mediciones basales antes de la intervención}

La edad y el sexo fueron consignados según la ficha médica del paciente.

La presión arterial sistólica (PAS), y la presión arterial diastólica (PAD) se midieron con un aparato de presión automático digital y se expresó en milímetros de mercurio $(\mathrm{mmHg})$. La presión arterial media (PAM) se calculó con la siguiente fórmula: PAM= PAD + (PAS $\mathrm{PAD}) / 3$.

La frecuencia cardíaca se obtuvo contando el número de latidos cardíacos, auscultados con un estetoscopio precordial durante un minuto.

La frecuencia respiratoria se obtuvo contando el número de excursiones torácicas durante un minuto.

Las mediciones de la PAS, PAD, frecuencia cardíaca y frecuencia respiratoria fueron realizadas con el paciente en posición decúbito dorsal y luego de 5 minutos de reposo.

\section{Mediciones después de la intervención}


Cambio de las cifras de PAM al ingresar a quirófano. Variable contínua. El cambio de las cifras de presión arterial resultó de la diferencia entre las cifras de presión arterial medidas la noche previa a la cirugía y las cifras de presión arterial medidas al ingresar al quirófano para la cirugía. Fue expresado con valores positivos si existió un aumento y valores negativos si hubo descenso de las cifras comparando ambas mediciones.

Cambio de la cifras de frecuencia cardíaca al ingresar al quirófano. Variable discreta. Resultó de la diferencia entre la frecuencia cardíaca medida la noche anterior a la cirugía y la frecuencia cardíaca medida al ingresar al quirófano para la cirugía. Fue expresado con valores positivos si existió un aumento y valores negativos si hubo descenso de las cifras comparando ambas mediciones.

Cambio en la frecuencia respiratoria al ingresar al quirófano. Variable discreta. Resultó de la diferencia entre la frecuencia respiratoria medida la noche anterior a la cirugía y la frecuencia respiratoria medida al ingresar al quirófano para la cirugía. Fue expresado con valores positivos si existió un aumento y valores negativos si hubo descenso de las cifras comparando ambas mediciones.

Las mediciones en quirófano luego de la intervención se realizaron en las mismas condiciones que antes de la intervención por un investigador que no conocía a que grupo pertenecían los pacientes.

\section{Asuntos estadísticos}

La hipótesis alternativa de estudio asumía que la presión arterial media en quirófano aumenta significativamente en pacientes hipertensos programados para cirugías electivas sin pre-medicación con alprazolam por vía oral.

El tamaño de la muestra en este estudio con una variable predictiva dicotómica y una variable resultante continua se calculó teniendo en cuenta que el riesgo de mortalidad cardiovascular se duplica con cada incremento de $20 \mathrm{mmHg}$ en la PAS y $10 \mathrm{mmHg}$ en la PAD (13-15) equivalente a incrementos de $13,3 \mathrm{mmHg}$ en las cifras de PAM y con el interés de detectar al menos una reducción deseable del $50 \%$ de los valores que duplican el riesgo cardiovascular en los pacientes. Llevando los datos a tablas estadísticas (16) se estimó un número mínimo de 44 pacientes para cada grupo.

Los datos fueron cargados en el programa de Epi-Info 5.3. Los datos obtenidos fueron procesados con estadística analítica en el programa Epi-Info 5.3 y SPSS utilizándose la Prueba $t$ de Student para el análisis en base a la combinación de variable predictora dicotómica y resultantes continuas, considerándose significativos valores de $\mathrm{p}$ menores a 0,05 .

\section{Asuntos éticos}

En lo que respecta al alprazolam, ésta es una benzodiacepina ampliamente utilizada para los trastornos de ansiedad por lo que no existen riesgos relevantes suscitados con su administración por vía oral a las dosis utilizadas en este trabajo (17). Así, la administración de alprazolam como medicación preoperatoria podrá beneficiar a los pacientes disminuyendo el grado de ansiedad y evitando el aumento de la presión arterial preoperatoria e incluirse en protocolos de pre-medicación.

Con respecto al conflicto de intereses no se contó con ningún laboratorio ni patrocinador involucrado y los gastos fueron afrontados por los investigadores.

\section{RESULTADOS}

Participaron de estudio un total de 88 pacientes, de los cuales el $72,7 \%$ (64 pacientes) fue de sexo femenino y el $27,3 \%$ (24 pacientes) del sexo masculino.

En la Tabla 1 se muestran la distribución de los grupos de pacientes y la presión preoperatoria, no existiendo diferencias significativas en la distribución de los pacientes hipertensos en ambos grupos. 
Tabla 1. Distribución de los pacientes en cada grupo $(n=88)$.

\begin{tabular}{|c|c|c|c|}
\hline & Grupo A $(n=44)$ & Grupo P $(n=44)$ & Valor $\mathrm{p}$ \\
\hline Sexo & & & 1 \\
\hline Femenino & 32 & 32 & \\
\hline Masculino & 12 & 12 & \\
\hline Edad media (años) & 53,8 & 54,3 & 0,78 \\
\hline $\begin{array}{l}\text { Niveles de presión arterial antes de la } \\
\text { medicación }\end{array}$ & & & 0,75 \\
\hline Normotensión y Prehipertensión & 15 & 13 & \\
\hline HTA 1 y HTA 2 & 29 & 31 & \\
\hline $\begin{array}{l}\text { Media de la PAM antes de la medicación } \\
(\mathrm{mmHg})\end{array}$ & 108,6 & 106,8 & 0,34 \\
\hline
\end{tabular}

En los pacientes que recibieron vitamina $C$ se constató un aumento significativo de la PAM en el quirófano en comparación con los que recibieron alprazolam como se observa en la Figura 1.



Figura 1. Variaciones de la PAM en los pacientes hipertensos seleccionados $(n=88)$ entre la toma del día anterior a la cirugía y la toma en quirófano, en ambos grupos.

En el grupo $A(n=44)$ se encontró una variación promedio de la PAM de $0,27 \mathrm{mmHg}(-$ 3,29 a 3,83), mientras que en el grupo $P(n=44)$ la variación promedio de la PAM fue de $7,59 \mathrm{mmHg}(4,4$ a 10,77$)$. Se compararon las medias de las variaciones en la PAM obteniéndose diferencias significativas ( $p=0,0035$; prueba t de Student).

Con respecto a los niveles de presión arterial de los pacientes hipertensos seleccionados, cuando fueron evaluados el día anterior a la cirugía programada, el 2,3\% $(n=2)$ presentó 
normotensión, el 29,5\% $(n=26)$ prehipertensión, el 38,6\% $(n=34)$ hipertensión grado 1 y el $29,5 \%(n=26)$ hipertensión grado 2 .

Tomando el grupo A $(n=44)$, según el estado de la presión arterial en el preoperatorio (prehipertensión, hipertensión grado 1 e hipertensión grado 2) y hallando el promedio de las variaciones de la PAM en dichos pacientes se encontró que los prehipertensos $(n=15)$ presentaron una variación media de la PAM de $4,6 \mathrm{mmHg}(1,35$ a 7,84), los hipertensos grado $1(n=14)$ presentaron $1,5 \mathrm{mmHg}(-3,58$ a 6,58$)$ de variación promedio y los hipertensos grado $2(n=15)-5,2 \mathrm{mmHg}(-9,21$ a $-1,18)$. Se observó diferencia significativa entre dichas medias (ANOVA), por lo que afirmamos que los pacientes que se premedicaron con alprazolam varían su PAM según el estado de la presión arterial en el preoperatorio.

Tomando el grupo $P(n=44)$, según el estado de la presión arterial en el preoperatorio (prehipertensión, hipertensión grado 1 e hipertensión grado 2), los prehipertensos $(n=11)$ presentaron una variación media de la PAM de $7,35 \mathrm{mmHg}(7,34$ a 9,75$)$, los hipertensos grado $1(n=20)$ una variación de $6,2 \mathrm{mmHg}(2,7$ a 9,69) y los hipertensos grado $2(n=11)$ $5,54 \mathrm{mmHg}(1,89$ a 9,18$)$. No se hallaron diferencias significativas entre las medias (ANOVA). Dos pacientes del grupo $P$ estuvieron normotensos el día anterior y se excluyeron de esta comparación por representar un número muy pequeño de pacientes.

Estadificando según el sexo, en el grupo A se obtuvo una variación promedio de la PAM en el sexo femenino de $-0,46 \mathrm{mmHg}(-3,71$ a 2,8$)$ y en el sexo masculino $2,25 \mathrm{mmHg}(0,94$ a 5,44 ). Estos valores no fueron significativamente estadísticos, $p=0,51$ (Prueba $t$ de student). En el grupo $P$, el sexo femenino presentó una variación promedio de la PAM de $6,75 \mathrm{mmHg}(4,15$ a 9,34$)$ y el sexo masculino $9,83 \mathrm{mmHg}$. $(5,25$ a 14,4$),(p=0,4)$. No se notaron diferencias significativas en las variaciones de la PAM según el sexo del paciente en los que consumieron alprazolam ni en los que consumieron vitamina $\mathrm{C}$.

En los pacientes que recibieron vitamina $C$ se constató un aumento significativo de la frecuencia cardíaca en el quirófano en comparación a los que recibieron alprazolam como se puede ver en la Figura 2.

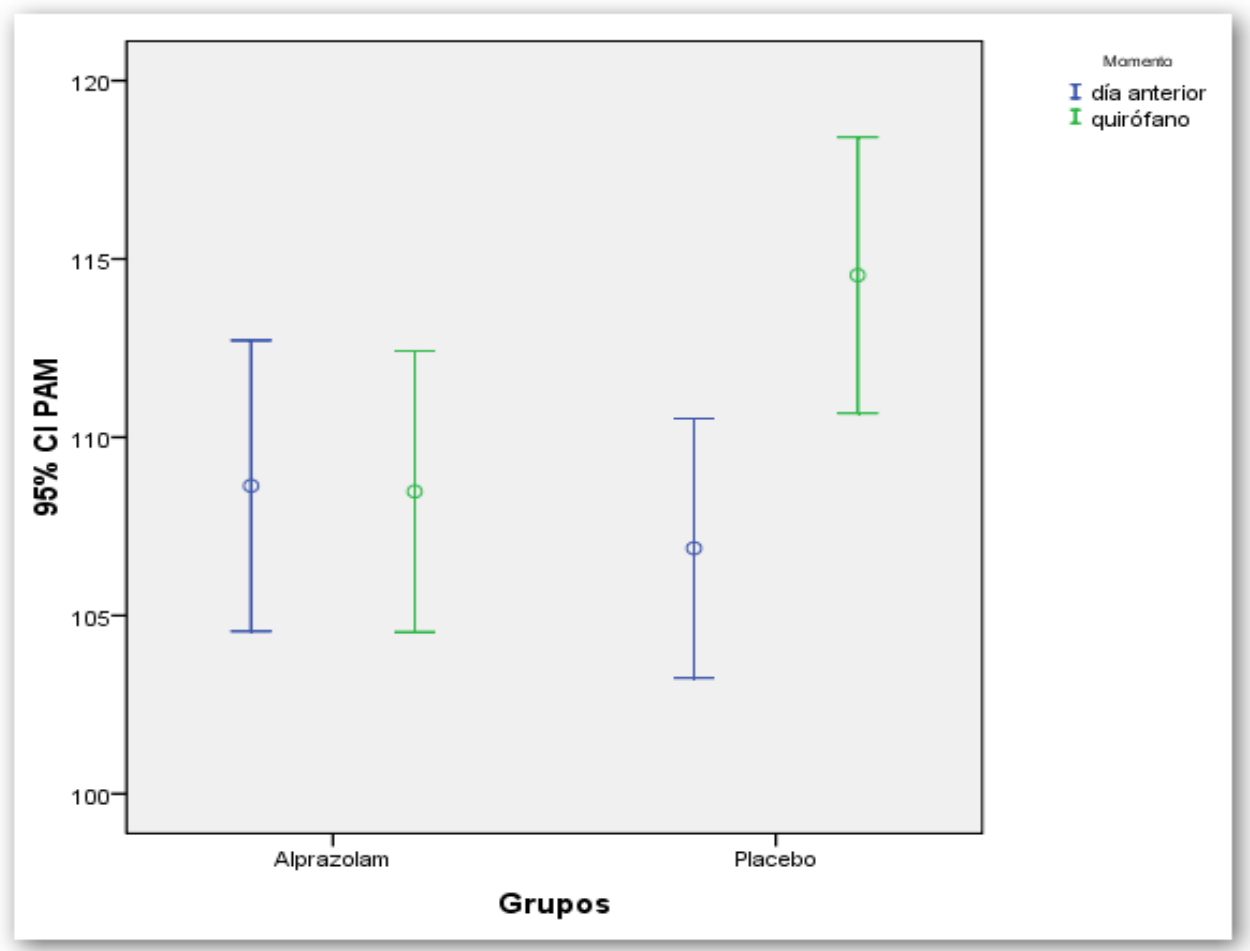

Figura 2. Variación de la frecuencia cardíaca en los pacientes hipertensos seleccionados $(\mathrm{n}=88)$ entre la toma del día anterior a la cirugía y la toma en quirófano, en ambos grupos

En el grupo A $(n=44)$ se encontró una variación promedio de la frecuencia cardíaca (FC) de $-2,1363$ latidos por minuto $(-5,327$ a 1,054$)$, mientras que en el grupo $P(n=44)$ la variación promedio de la FC fue de 5,38 latidos por minuto $(2,59$ a 8,16). Comparándolas, 
estas medias de las variaciones en la FC pudo constatárse diferencias estadísticamente significativas en ambos grupos ( $p=0,0008$ Prueba $t$ de Student).

Con respecto a la variación de la frecuencia respiratoria, no se observaron diferencias entre los que se premedicaron con alprazolam y con placebo como se puede ver en la Figura 3.

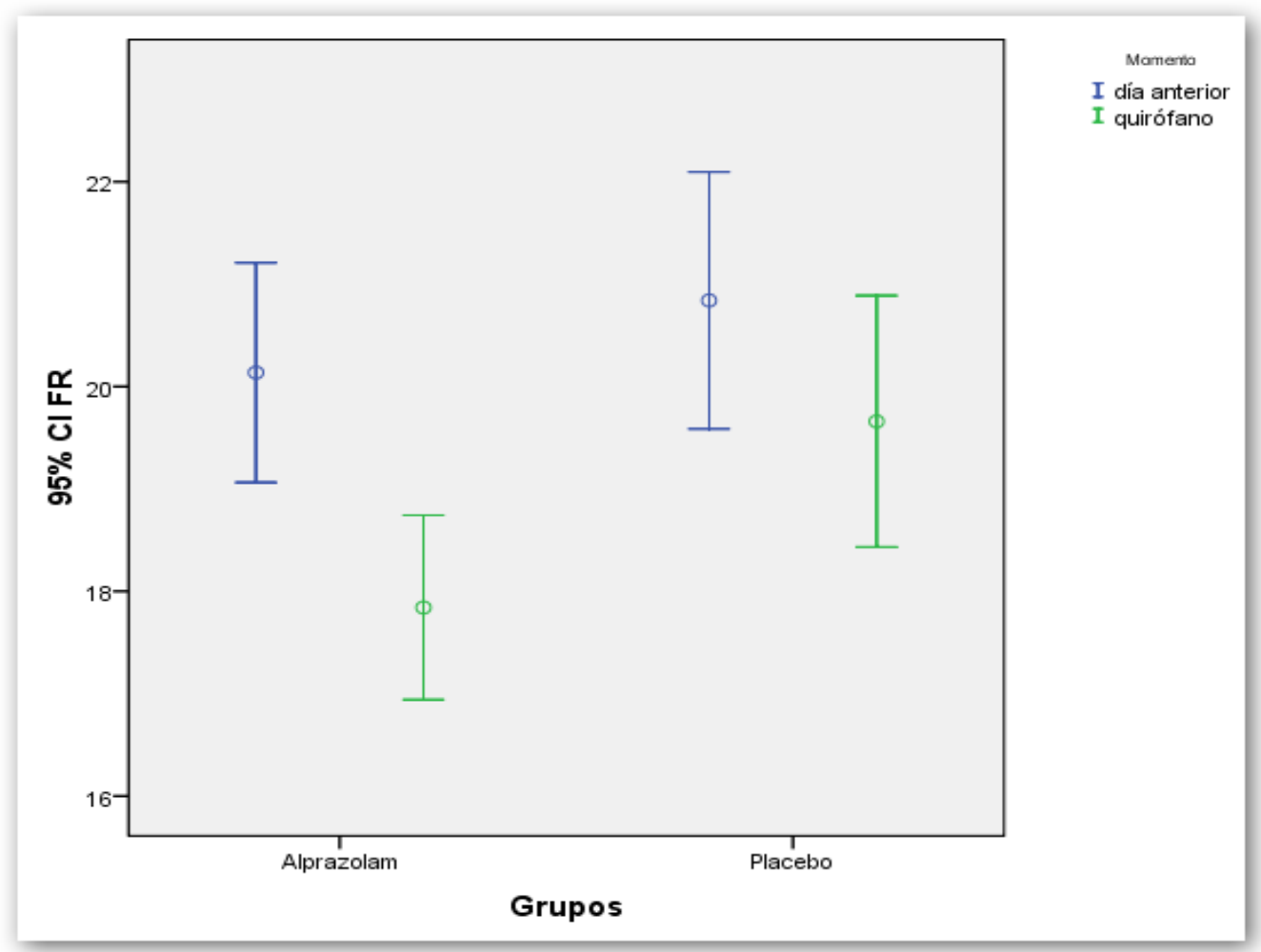

Figura 3. Variación de la frecuencia respiratoria en los pacientes hipertensos seleccionados $(n=88)$ entre la toma del día anterior a la cirugía y la toma en quirófano.

En el grupo A $(n=44)$ se encontró una variación promedio de la frecuencia respiratoria (FR) de $-2,29$ respiraciones por minuto $(-3,36$ a $-1,21)$, mientras que en el grupo $P(n=44)$ la variación promedio de la FR fue de $-1,18$ respiraciones por minuto $(-2,51$ a 0,15$)$. Comparando las medias de las variaciones en la FR mediante la Prueba t de Student, $(p=$ $0,206)$, no se hallaron diferencias estadísticamente significativas en ambos grupos con respecto a la variación de la frecuencia respiratoria.

\section{DISCUSIÓN}

La ansiedad en el período preoperatorio es un hecho prácticamente constante hasta el momento de la intervención, desencadenando en el organismo una respuesta al estrés, con descarga de catecolaminas e hiperactividad simpática (18). Esta situación pasa a ser muy relevante en los pacientes hipertensos ya que poseen de base una alteración en el control de la presión arterial.

Este estado de ansiedad puede repercutir en el curso evolutivo del periodo intraoperatorio de la anestesia de manera que se puede producir una mayor incidencia de inestabilidad hemodinámica, o requerimiento de mayores dosis de fármacos (19), por lo que resulta conveniente prever este factor para minimizar su influencia, lo que se lograría con la premedicación con alprazolam.

Varios estudios sobre diferentes tipos de intervenciones pre-anestésicas o prequirúrgicas han encontrado múltiples causas que pueden generar este tipo de estrés emocional previo al acontecimiento anestésico como lo son el miedo de los pacientes al ambiente hospitalario, la preocupación por la anestesia y la cirugía, también por el diagnóstico postoperatorio, la posibilidad de despertar en el intra-operatorio o de no despertar al final del procedimiento, el temor al dolor postoperatorio, desconocimiento del cirujano y de la cirugía misma (19- 
21). A medida que se acerca la hora marcada para la cirugía, el pensamiento en estos factores hace que se acreciente la reacción de estrés del paciente.

A pesar de esto, los pacientes deben estar compensados al ser internados para una cirugía electiva o de lo contrario debe diferirse la fecha de cirugía hasta alcanzar un control adecuado de las comorbilidades. Llama la atención de que varios de los pacientes hipertensos ingresados para cirugías electivas no se encontraban compensados adecuadamente, ya que al ser controlados el día anterior a la cirugía en la sala de internación, más de la mitad de los mismos (60 pacientes) presentaron hipertensión de grado 1 o grado 2 . Este tema amerita un estudio más profundo ya que idealmente para una cirugía electiva estos pacientes deben encontrarse bien controlados manteniendo presiones menores a $140 / 90 \mathrm{mmHg}(22)$, evitando contratiempos como suspensiones de las cirugías en el quirófano, descompensaciones clínicas 0 aumento de las complicaciones peroperatorias y postoperatorias.

Algunos estudios señalan que en el quirófano la ansiedad de los pacientes pre-medicados con benzodiacepinas fue significativamente menor que la de los pre-medicados con drogas no benzodiacepínicas o con placebo como sostienen Onarque et al. y De Vite et al. (2325). En este estudio se encontró que los valores de la PAM variaron mínimamente en los pacientes hipertensos que recibieron alprazolam $(0,27 \mathrm{mmHg})$ como medicación preoperatoria, mientras que se evidenció un aaumento significativo de los valores de la PAM en los pacientes que no recibieron alprazolam $(7,59 \mathrm{mmHg})$. La diferencia de $7,32 \mathrm{mmHg}$ entre ambos grupos refleja la contribución del alprazolam a evitar tan significativo aumento de la PAM y constatándose una contribución de este fármaco en la minimización de algunos factores que desencadenan el aumento de la presión arterial en estos pacientes, como lo son la ansiedad y nerviosismo preoperatorio (26).

Para individuos entre 40 y 70 años de edad, cada incremento de $20 \mathrm{mmHg}$ en presión sistólica o $10 \mathrm{mmHg}$ en presión diastólica dobla el riesgo de eventos cardiovasculares a partir de cifras tensiónales de $115 / 75 \mathrm{mmHg}$ a $185 / 115 \mathrm{mmHg}$ (13). Esto equivale a incrementos de $13,3 \mathrm{mmHg}$ en la PAM. La relación entre presión arterial y eventos cardiovasculares es continua, consistente e independiente de otros factores de riesgo, a mayor cifras de presión arterial mayor riesgo de infarto de miocardio, insuficiencia cardiaca, accidentes cerebrovasculares y enfermedad renal (27). Con la pre-medicación en el periodo preoperatorio con alprazolam se consiguió evitar un aumento promedio de $7,32 \mathrm{mmHg}$ en la PAM contribuyendo a reducir las posibilidades de incrementos tensionales que pudiesen aumentar los riesgos de complicaciones.

En los hipertensos que fueron medicados con vitamina $C$ no hubo diferencias en las variaciones de la PAM, según el estado de la misma que presentaban al momento de internarse, pero el hallazgo del grado de modificación de la PAM producida por el alprazolam se relacionó con los niveles de PAM que presentaron los pacientes en la sala de internación. El efecto de disminución de la PAM con alprazolam fue evidente en los pacientes internados con hipertensión grado 2, en comparación a los demás pacientes. En los hipertensos grado 1 hubo un ligero aumento y en los prehipertensos un mayor aumento de la PAM. Esto denota una mayor contribución del efecto del alprazolam en los pacientes con cifras de presión arterial más elevadas.

En los pacientes medicados con alprazolam se produjo una ligera disminución de la frecuencia cardíaca en comparación a los que fueron medicados con placebo, en los que no aumentó la frecuencia cardíaca. El aumento de la frecuencia cardíaca puede ser perjudicial en pacientes con cardiopatías como por ejemplo en los pacientes coronarios, ya que aumenta el consumo de oxígeno miocárdico y puede descompensar a estos pacientes. Además, el aumento de la frecuencia cardiaca puede incrementar el volumen cardíaco, pero a costa de una reducción de la eficiencia; esta situación puede ser bien tolerada si el aporte de flujo coronario es adecuado, pero en caso de estenosis de las arterias coronarias, el efecto del aumento en la frecuencia cardiaca es deletéreo (28). Con la administración de alprazolam se evitaría un aumento significativo de la frecuencia cardíaca y beneficiaría a los pacientes hipertensos con cardiopatía isquémica.

Debe considerarse la protocolización de la pre-medicación ansiolítica, en especial en los pacientes hipertensos, ya que estos son más sensibles a las catecolaminas liberadas en mayor cantidad durante el pre-operatorio como consecuencia estrés (29). La minimización de la ansiedad con la contribución farmacológica del alprazolam puede redituar en que el paciente llegue a quirófano con un control adecuado de la presión arterial y la frecuencia cardíaca. Se evitarían problemas prevenibles como la suspensión de la cirugía por crisis 
hipertensiva, la descompensación peroperatoria o las complicaciones postoperatoria de las comorbilidades.

En conclusión, utilizando alprazolam por vía oral como medicación en el periodo preoperatorio se evitaron aumentos significativos de la presión arterial y de la frecuencia cardíaca en los pacientes hipertensos programados para cirugías electivas incluidos en este estudio, por lo que es pertinente su utilización como medicación preoperatoria para cirugías electivas en los pacientes hipertensos.

\section{REFERENCIAS BIBIOGRÁFICAS}

1. Dix P, Howell S. Survey of cancellation rate of hypertensive patients undergoing anaesthesia and elective surgery. $\mathrm{Br}$ J Anaesth. 2001;86:789-93

2. Rose D, Marsha C, DeBoer P. Cardiovascular events in the postoperative care unit: Contribution of risk factors.Anesthesiology.1996;84:772-8

3. Chung F, Mezei G, Tong D. Pre-existing medical conditions as predictors of adverse events in day case surgery. $\mathrm{Br}$ J Anaesth. 1999; 83:262-70

4. Howell S, Sear Y, Yeates D, Goldcare M, Sear J, Foex P. Risk factors for cardiovascular death after elective surgery under general anaesthesia. $\mathrm{Br} \mathrm{J}$ Anaesth.1998;80:14-9

5. Bavry $T$, Coriat $P$. Hypertension and anaesthesia. EMC- Anesthèsie Réanimation 2004; 1 : 25-53.

6. Ruiz-López E, Muñoz-Cuevas JH, OliveroVásquez YI, Islas-Saucillo M. Preoperatory anxiety at the General Hospital of Mexico. Rev Med Hosp Gen Mex 2000;63:231-36

7. Valenzuela J, Barrera J, Ornella J. Ansiedad preoperatoria en procedimientos anestésicos. Cir Cir 2010;78:151-56

8. Kiyohara $L$, Kayano $L$, Oliveira $L$, Yamamoto $\mathrm{M}$, Inagaki $\mathrm{M}$, Ogawa $\mathrm{N}$, et al. Surgery information reduces anxiety in the pre-operative period. Rev Hosp Clin Fac Med Sao Paulo 2004;59:51-6

9. Kain Z, Sevarino $F$, Rinder C, Pincus $S$, Alexander G, Ivy $M$, Heninger $G$. Preoperative anxiolysis and postoperative recovery in women undergoing abdominal hysterectomy. Anesthesiology 2001; 94:415-22

10. Kil H, Kim W, Chung W, Kim G, Seo $H$, Hong J. Preoperative anxiety and pain sensitivity are independent predictors of propofol and sevoflurane requirements in general anaesthesia. $\mathrm{Br} J$ Anaesth. 2012;108(1):119-25

11. Grossman $E$, Nadler $M$, Sharabi $Y$, Thaler M, Shachar A, Shamiss A. Antianxiety treatment in patients with excessive hypertension. Am J Hypertens. 2005; 18:1174 - 77.

12. Yilmaz S, Pekdemir M, Tural U, Uygun M. Comparison of alprazolam versus captopril in high blood pressure: A randomized controlled trial. Blood Press. 2011;20: 239-43

13. Chobanian A, Bakris G, Black $H$, Cushman W, Green L, Izzo J, Jones D, et al. The Seventh Report of the Joint National Committee on Prevention, Detection, Evaluation, and Treatment of High Blood Pressure. JAMA 2003;289(19):2560-71

14. Lewington $S$, Clarke R, Quizilbash N, Peto R, Collins R. Age-specific relevance of usual blood pressure to vascular mortality: a meta-analysis of individual data for one million adults in 61 prospective studies. Lancet. 2002; 60:1903-13

15. Hiroki M, Kajimura N, Uema T, Ogawa K, Nishikawa K, Kato M, Watanabe T, et al. Effect of benzodiazepine hypnotic triazolam on relationship of blood pressure and $\mathrm{PaCO} 2$ to cerebral blood flow during human non-rapid eye movement sleep. J Neurophysiol 2006; 95:2293-303

16. Hulley S, Browner W, Newman T. Cálculo de la potencia y del tamaño de la muestra. In: Hulley $S$ (ed). Diseño de investigaciones clínicas. Lippincott Williams \& Wilkins. Philadelfia. 2008. pp 95

17. Hurlé M. Fármacos ansiolíticos y sedantes. In: Florez J (ed). Farmacología humana. 3ra ed. Masson. Barcelona. 1997. pp 455-64

18. Biebuyck JF The metabolic response to stress: an overview and update. Anesthesiology 1990; 308-27.

19. Maranets I, Kain ZN Preoperative anxiety and intraoperative anesthetic requirements. Anesth Analg 1999; 89: 1346-351.

20. Kindler C, Harms C. The Visual Analog Scale Allows Effective Measurement of Preoperative Anxiety and Detection of Patients' Anesthetic Concerns. Anesth Analg 2000; 90:706 -12.

21. Ruiz E, Muñoz J. Ansiedad preoperatoria en el Hospital general de México. Rev Med Hosp Gen Mex 2000; 63(4): 231-36

22. Fleisher L. Preoperative evaluation of the patient with hypertension. JAMA 2002; 287: 2043-6.

23. Ornaque I, Carrero E, Villalonga A, Roux C, Salvador L. Estudio de la ansiedad prequirúrgica, ginecológica y oftálmica en relación con la administración o no de premedicación ansiolítica. Rev Esp Anestesiol Reanim. 2000;47(4):151-6.

24. Franssen $\mathrm{C}$ et al. Comparison between alprazolam and hydroxyzine for oral 
premedication. Can J Anaesth. 1993;

40:13-7.

25. De Witte JL, Algret C, Sesler DI. Preoperative alprazolam reduces anxiety in ambulatory surgery patients: a comparison with oral midazolam. Anesthesia and Analgesia 2002; 95:1601-1606.

26. Player M, King D, Mainous A, Geesey M. Psychosocial Factors and Progression From Prehypertension to Hypertension or Coronary Heart Disease. Annals of Family Medicine. 2007; 5 : 403-11

27. Vasan R, Beiser A, Seshadri S, Larson M, Kannel W, D'Agostino N, Levy D. Residual lifetime for developing hypertension in middle-aged women and men: The Framingham Heart Study. JAMA 2002; 287:1003-10

28. Moreu-Burgosa J, Macaya C. Fisiopatología del miocardio isquémico. Importancia de la frecuencia cardiaca. Rev Esp Cardiol Supl. 2007;7:19-25

29. Fisiología del Estrés. Instituto Nacional de Seguridad e Higiene en el Trabajo. Ministerio de Trabajo y Asuntos Sociales. España. Disponible en: http://www.insht.es/InshtWeb/Contenid os/Documentacion/FichasTecnicas/NTP/F icheros/301a400/ntp_355.pdf 